\title{
Territoires et grandes vitesses en Europe
}

Table ronde animée par Gabriel Dupuy

Territories and high-speed trains in Europe

\section{Gabriel Dupuy, Etienne Auphan et Michel Walrave}

\section{OpenEdition}

\section{Journals}

Édition électronique

URL : https://journals.openedition.org/rhcf/875

DOI : 10.4000/rhcf.875

\section{Éditeur}

Rails \& histoire

\section{Édition imprimée}

Date de publication : 1 juin 2008

Pagination : 127-137

ISSN : 0996-9403

\section{Référence électronique}

Gabriel Dupuy, Etienne Auphan et Michel Walrave, «Territoires et grandes vitesses en Europe », Revue d'histoire des chemins de fer [En ligne], 39 | 2008, mis en ligne le 01 juin 2011, consulté le 22 avril 2022 URL : http://journals.openedition.org/rhcf/875 ; DOI : https://doi.org/10.4000/rhcf.875

Ce document a été généré automatiquement le 22 avril 2022.

Tous droits réservés 


\title{
Territoires et grandes vitesses en Europe
}

\author{
Table ronde animée par Gabriel Dupuy \\ Territories and high-speed trains in Europe
}

Gabriel Dupuy, Etienne Auphan et Michel Walrave

\section{Gabriel Dupuy}

J'ouvre une table ronde qui nous permet d'avoir à nos côtés Michel Walrave, grand témoin de l'histoire récente ferroviaire. Etienne Auphan et moi-même nous sommes mis d'accord sur une série de questions qui ouvriront le débat. Nous pourrons d'une part poser des questions relatives aux exposés précédents qui ont dressé le bilan des vingt ans d'études menées par l'AHICF et d'autre part lancer un débat à propos des interventions concernant des expériences de pays étrangers dans le domaine de la grande vitesse ferroviaire.

2 Je commence par poser la question de l'adaptation d'un système de grande vitesse ferroviaire à un territoire donné. José Maria de Ureña Francés a mis en lumière une différence substantielle entre la conception d'un réseau espagnol, national dans son organisation, et celle d'un réseau français qui semble bien vouloir s'européaniser. Du côté coréen", nous avons l'impression d'un territoire extrêmement différent du territoire français dans son occupation auquel pourtant un système technologique analogue au système français a su s'adapter.

\section{Michel Walrave}

Je remercie l'AHICF de son invitation et c'est toujours avec grand plaisir que je participe à ses colloques afin de confronter un point de vue technico- économique à l'analyse des historiens et aujourd'hui des géographes. En effet, dans cette grande histoire des réseaux, les déterminants de géographie humaine et les déterminants historiques sont fondamentaux ; on ne travaille pas à partir de rien, mais à partir d'une longue histoire qu'il faut transformer avec les moyens technologiques du moment. Dans son remarquable exposé, le professeur de Ureña Francés a insisté à juste titre sur les similitudes entre réseaux français et espagnol. Les débats précédents ont mentionné 
Paris et le désert français et il est vrai que la première ligne de TGV a traversé le fameux désert français de M. Gravier. Or on pourrait aussi parler de désert espagnol, parce que la géographie de l'Espagne distingue une très grosse agglomération, Madrid, quoiqu'elle n'ait pas la taille de l'agglomération parisienne, et une série de villes situées à la périphérie et dans toutes les directions. Cette configuration rend les choses beaucoup plus difficiles du point de vue de la conception des réseaux et du point de vue de l'économie du système parce que les flux sont forcément éventaillés dans toutes ces directions et que, avec quatre grands axes majeurs, il est difficile de couvrir certaines zones, en particulier le nord-ouest de l'Espagne. En France, avec quatre lignes radiales on parvient à peu près à couvrir toutes les directions ; en France (sans comparer son cas avec les densités humaines du Japon et de la Corée - la Corée c'est le corridor du Tokaido divisé par deux pour ce qui concerne la taille de Séoul et des agglomérations à relier, avec une distance presque similaire à la ligne japonaise) nous avons aussi la chance d'avoir l'agglomération parisienne, Lille, Lyon et Marseille presqu'alignés sur un même axe, et ce n'est pas le fruit du hasard si on a commencé par construire la ligne Paris-Lyon. Le TGV Atlantique a suivi à cause des avatars qui ont marqué le projet de TGV Nord et surtout de tunnel sous la Manche, qui a failli démarrer en 1975 mais est resté en plan une vingtaine d'années. Lorsqu'à l'origine on a discuté au sein de la SNCF pour choisir entre le TGV Nord et l'axe Paris - Sud-Est, ce dernier s'est imposé pour plusieurs raisons, d'une part à cause de la démographie, d'autre part à cause de la gamme des distances, plus propices à l'expression de la grande vitesse. Par ailleurs vers le Nord le projet n'avait de sens que s'il était international ; or lancer d'emblée un projet aussi innovant sous tous ses aspects que le TGV sur un plan international lorsqu'on connait les lenteurs et les difficultés, les contraintes de tous ordres qui marquent la coopération internationale encore plus grandes à l'époque qu'à présent, semblait déraisonnable : il fallait pour concevoir un système avoir les coudées franches et rester " entre nous ", c'est l'un des éléments qui ont conduit au choix de l'axe Paris - Sud-Est.

Un autre grand débat, au début du TGV, était l'exemple japonais; les études du TGV avaient démarré en 1966-1967 et le Japon venait de mettre en service la ligne du Tokaido en 1964. Évidemment, beaucoup de gens disaient «le Japon n'est pas la France » (le corridor du Tokaido, 50 millions d'habitants, dont la moitié à Tokyo, concentrait à lui seul la population de toute la France), et les données de territoire, géographie et démographie, étaient donc fondamentalement différentes. On dit parfois que la pauvreté rend intelligent et nous, face à la pauvreté des trafics potentiels en comparaison avec les énormes trafics du Japon, nous avons conçu un système dont la ligne directrice était l'économie de moyens, l'économie de construction de l'infrastructure, l'économie du matériel, l'économie de l'exploitation, en visant notamment des coefficients d'utilisation du matériel très élevés, jugés très irréalistes à l'époque mais dont l'expérience a montré qu'ils pouvaient encore être dépassés par les nouvelles politiques commerciales du type yield management.

5 Si je reviens à l'Espagne, nous avons cette différence de la dispersion géographique espagnole ; le fait aussi qu'en France nous avons plus de chance du point de vue du relief : l'Espagne a des zones difficiles à franchir au nord de Madrid et vers l'Andalousie, il faut donc des tunnels importants, ouvrages d'art que nous avons pu éviter sur la première ligne et celles qui ont suivi. Du point de vue des caractéristiques orographiques du territoire, l'Espagne est ainsi beaucoup plus pénalisée que la France. Deux différences ont été évoquées sur lesquelles il faut revenir. Bien sûr, la différence 
dans la réalisation de l'objectif de compatibilité des circulations sur les lignes nouvelles et les lignes existantes, puisqu'en Espagne la différence d'écartement est une sujétion très importante. Il faut vraiment saluer la vision d'avenir du gouvernement espagnol de l'époque qui, en privilégiant l'aspect européen et la vision à long terme, a choisi de donner à la ligne Madrid-Séville l'écartement standard européen malgré les problèmes d'exploitation très importants que cela pose à court terme et que l'Espagne va résoudre progressivement, au prix quand même de grandes difficultés et de controverses quant aux choix effectués.

6 Je crois aussi que, du fait de la géographie espagnole, l'objectif du maillage du réseau est économiquement beaucoup plus difficile à réaliser en Espagne qu'en France où nous avons déjà des transversales assez importantes, comme l'axe Strasbourg-vallée du Rhin-vallée du Rhône, ou la vallée de la Garonne, le Languedoc ; il y a déjà des transversales avec des agglomérations situées dans des gammes de distance telles que le train à grande vitesse puisse y jouer un rôle important. Autre différence de poids, c'est que le réseau français [atteint une certaine rentabilité ] - je mets à part le cas du TGV Est, typiquement un TGV d'aménagement du territoire et d'ouverture vers l'Europe - alors que l'Espagne, finalement, et c'est aussi un choix très courageux du gouvernement espagnol, a investi à fonds perdus dans la construction de l'infrastructure. On parvient à l'équilibre financier de l'exploitation, mais l'infrastructure ne peut pas être rentabilisée à cause de la faiblesse des courants de trafic.

7 Enfin deux différences qui ont été évoquées. La différence de qualité : l'AVE espagnol obtient des performances de régularité excellentes et celles de la SNCF sont nettement moins bonnes. Quand nos amis espagnols ont mis en place cette mesure commerciale qui consistait à rembourser le prix du voyage pour tous les trains dont le retard excédait cinq minutes, nous étions un peu inquiets car cela était de nature à susciter des demandes similaires en France. Or celles-ci sont impossibles à satisfaire ; c'est le revers de la médaille de la compatibilité : la compatibilité présente l'avantage de desservir sans rupture de charge toutes les gares situées en dehors des lignes à grande vitesse mais, à l'inverse, elle expose le trafic à grande vitesse à tous les aléas de l'exploitation sur le réseau classique. Alors que, quand on a une exploitation de densité plus faible, comme c'est le cas en Espagne, abritée de ces sujétions, on peut évidemment viser et obtenir des performances de régularité bien meilleures. Dernière différence, celle de la politique tarifaire : l'AVE espagnol a été conçu davantage comme un train de luxe même s'il a une classe touriste ouverte à tous, avec une tarification nettement supérieure à celle des trains conventionnels alors que la politique française a été de viser d'emblée le transport de masse avec une politique tarifaire très modérée qui à mon avis en a fait le succès non seulement économique mais aussi populaire et politique comme l'a rappelé madame Idrac.

\section{Gabriel Dupuy}

Je demande l'avis du géographe sur la même question...

\section{Etienne Auphan}

Je crois que le modèle français à grande vitesse, le premier modèle européen du genre, au sens global du terme, et qui a reçu en premier son application territoriale, a été conçu dans le cadre d'un territoire bien déterminé, celui de la France, avec une technologie qui s'est adaptée à ce territoire, et j'ai la conviction qu'on ne peut pas séparer le modèle français à grande vitesse du territoire pour lequel il a été conçu. La 
question qui s'ensuit alors est celle de savoir dans quelle mesure ce modèle peut être adapté à des territoires autres, étant entendu qu'on supposait que la technologie pouvait être exportée sans problème : le défi principal est de savoir si, en dehors de la technologie, l'application territoriale pouvait s'opérer dans des territoires différents. Michel Walrave l'a parfaitement rappelé : ce modèle a été fondé sur le principe visant à construire la plus faible longueur d'infrastructure nouvelle pour assurer le plus grand nombre de liaisons possibles. Il y a là en France quelque chose d'unique en son genre puisqu'autant en Corée qu'au Japon les lignes nouvelles sont des corridors et qu'en Espagne on hésite entre un schéma radial et un schéma maillé. Les quatre axes radiaux français correspondent à un besoin, à un problème économique très précis qui est de construire la plus faible longueur d'infrastructure.

10 Deuxième point, ce modèle, précisément, a engendré la massification des flux sur le modèle aérien du hub, même s'il ne s'agit que d'une assimilation imparfaite au concept de hub, le hub francilien, soit au sens strict avec transit à Paris, soit le hub fonctionnel mais non réel avec les liaisons province-province par l'interconnexion francilienne. Autre point, avec le modèle français à grande vitesse, il s'agit bien du premier exemple du passage d'une logique nationale de service public de desserte à une logique d'entreprise. Anne-Marie Idrac a évoqué dans ce colloque les impératifs de l'entreprise et je considère pour ma part que, dès le début des années 1980 et dans la conception même du TGV à la fin des années 1970, il s'agissait bien du premier exemple de passage à la logique d'entreprise dans lequel la dimension de desserte territoriale venait au deuxième plan ; la preuve, c'est précisément que le territoire français permettait en quelque sorte, mais moyennant des conséquences relativement importantes, Michel Walrave l'a rappelé, d'assurer quasiment toutes les relations importantes de ville à ville en France, qu'elles soient radiales ou territoriales, en s'affranchissant de cette zone topographiquement contraignante qui est au centre du territoire français, à savoir le Massif central. Donc on contourne l'obstacle, et on le contourne précisément par l'îlede-France qui se trouve décentrée au Nord. Dernier aspect, cette logique d'entreprise vient en porte-à-faux avec les contraintes de l'aménagement du territoire entendu d'une certaine manière en France, c'est-à-dire au sens d'équilibre territorial. On peut se référer sur ce point au schéma des liaisons ferroviaires à grande vitesse de 1992, aujourd'hui complètement dépassé car une grande partie est caduque du fait de sa dimension " politicienne ", mais dont il reste néanmoins une autre partie. Il y a là une confrontation entre les effets territoriaux de la mise en application du schéma des lignes à grande vitesse conçu par la SNCF et la demande politique devant les premiers succès de ses applications territoriales. Donc je pense que, vis-à-vis de la Corée et du Japon, il faut faire la différence entre la technologie, exportée dans le cas de la Corée, et le modèle territorial et je ne suis pas certain que le fait que la conception du modèle français associe une technologie et un territoire bien précis ait été parfaitement perçu dans les exportations du modèle.

\section{Gabriel Dupuy}

Une autre question : quand on se promène à Barcelone aujourd'hui, on y voit des grands chantiers ferroviaires. L'on nous explique qu'il s'agit de faire des gares de passage des trains à grande vitesse en plein cœur de la ville. Cela pose la question plus générale de l'interconnexion. En France cette question n'est pas spécifiquement parisienne puisqu'elle s'est posée également à Lille : c'est tout simplement la question de l'organisation du réseau. 
Un point très intéressant a été souligné par José Maria de Ureña Francés, c'est la possibilité de traverser les grandes agglomérations, Madrid ou Barcelone, par des tunnels ferroviaires existant ou à compléter. C'est en effet un avantage considérable. La ligne d'interconnexion faite en Île-de-France est encore une fois une conception économique. Elle était infiniment moins coûteuse que la construction d'une grande jonction sous Paris, qui se fera peut-être un jour, l'avenir n'est écrit nulle part... Une telle interconnexion, comme il en existe également à Bruxelles ou à Berlin, est un élément très intéressant pour le développement des réseaux et pour la desserte des grandes agglomérations. L'interconnexion en Île-de-France a visé un double objectif : d'une part, créer des relations de région à région passant par la région parisienne mais sans changements de train et surtout de gare à Paris (c'est une idée qui avait été évoquée par une association d'usagers des transports mais qui s'était révélée irréalisable avec des trains classiques car les temps de parcours et les flux de trafic n'auraient pas permis de remplir convenablement de tels trains), l'interconnexion, qui joue un rôle complémentaire par rapport aux lignes radiales, permettant de faire bénéficier à moindre coût ces trafics de région à région de tout l'investissement qui, lui, a été justifié par les grands flux radiaux ; d'autre part, de procurer un accès plus aisé à la grande vitesse pour les zones d'île-de-France, plus périphériques, sans même parler de l'atout majeur d'organiser une complémentarité avec le transport aérien à partir de Roissy.

13 Je voudrais faire une incidence : je ne suis pas tout à fait d'accord avec ce qu'a dit le professeur Auphan du schéma directeur de 1992. C'est peut-être un plaidoyer pro domo car je suis largement responsable de ce schéma, mais je ne crois pas qu'il soit dépassé. L'essentiel de ce schéma est en train de se concrétiser, toutes les lignes qui ont été réalisées et celles qui sont actuellement « dans les tuyaux » y figurent, et, par ailleurs, s'il existait quelques scories "politiques ", elles sont restées très limitées par rapport au schéma d'ensemble ; enfin, ce schéma fut la première occasion à partir de laquelle on a vraiment parlé d'ouverture européenne comme l'a montré la carte du professeur de Ureña avec les maillons qui prolongent les lignes nationales existantes vers le reste de l'Europe, notamment vers l'Europe du Nord et de l'Est.

J'ajoute à propos de cette interconnexion que sa réalisation a été anticipée de manière en quelque sorte accidentelle. Les demandes, voire les exigences d'Aéroports de Paris et d'Air France qui, craignant de voir leur trafic international de la région parisienne détourné sur l'aéroport de Bruxelles, avaient souhaité fortement le passage du TGV Nord dans l'aéroport de Paris (ce qui aurait coûté beaucoup de temps et beaucoup d'argent) ont indirectement déclenché la réalisation et surtout l'accélération de la mise en œuvre de l'interconnexion. À l'époque nous envisagions l'interconnexion à l'horizon 2010 et, en fait, sa réalisation a été beaucoup plus rapide car, prenant la balle au bond, nous avons dit à ADP et Air France, dont les demandes avaient reçu un fort soutien politique : "Plutôt que de nous pénaliser sur le TGV Nord et de vous donner seulement l'accès au nord de la France, en réalisant toute l'interconnexion nous vous offrons l'accès de Roissy à toutes les grandes métropoles de France. » C'était donc une réussite, du point de vue du territoire et de la complémentarité avec les autres modes de transport, un élément qui prend beaucoup de poids dans le schéma - complémentarité entre transport aérien et transport ferroviaire, avec les transports régionaux et avec les transports urbains. Je crois que ceci était tout à fait fondamental. 


\section{Etienne Auphan}

Une réflexion sur cette interconnexion francilienne qui résulte de la conjonction d'intérêts qui a priori n'étaient pas tous présents au départ et n'allaient pas dans le même sens. On vient de dire ce qu'il en est de Roissy, en rappelant précisément qu'initialement la desserte de Roissy CDG était prévue sur le TGV Nord et non sur l'interconnexion, pensée en tant que telle ; est venue ensuite l'opportunité d'Eurodisney, avec la participation d'Eurodisney à la desserte de Marne-la-Vallée, et puis, en dernier lieu, l'opportunité politique de Massy, d'une manière un peu marginale, avec la demande, la démarche très volontariste, du maire de l'époque, Claude Germon ; c'est ainsi qu'on en est arrivé à cette conjonction d'intérêts divers et de celui de la SNCF ; est venu également s'ajouter l'intérêt des responsables de l'aménagement francilien avec le fait que ces trois gares périphériques correspondaient en quelque sorte au développement en tache d'huile de l'agglomération parisienne, donc à la décongestion des gares centrales parisiennes, etc. ; sur ce plan-là, on peut dire que le contournement de l'île-de-France, l'interconnexion francilienne des TGV, résulte d'une ambiguïté très grande dans sa fonction, d'autres diraient de la conjonction des intérêts. En effet, elle donne à la fois la possibilité, soit directement, soit par correspondance, d'assurer des relations de province à province transversales autrefois assurées soit par la correspondance inter-gares parisiennes, soit par les liaisons directes inter-régionales transversales dans le centre et la moitié sud de la France mais aussi, par les mêmes TGV, la desserte périphérique des pôles d'activité parisiens, donc la desserte de Paris qui était assurée autrefois en partie ou en totalité par les gares centrales parisiennes et le RER vers la périphérie. Je crois pour ma part que cette ambiguïté se traduit encore actuellement par quelque chose qui n'est pas tout à fait satisfaisant sur le plan du fonctionnement territorial - c'est ici le géographe qui parle puisque cela continue à alimenter (on va me dire que c'est la constante du fonctionnement territorial français et qu'on ne peut faire autrement) la concentration des activités sur la région francilienne et contribue d'une certaine manière à renforcer le déséquilibre territorial qui est un élément important du débat. Voilà ce que je voulais dire sur l'ambiguïté fondamentale de cette interconnexion.

\section{Gabriel Dupuy}

Une autre question, évoquée par nos collègues, est celle de la spécialisation des trafics. Je pense notamment, dans le cas espagnol, au fait qu'il ne semble pas y avoir eu de choix entre fret et voyageurs, les deux registres étant liés, quelles que soient les difficultés. Par ailleurs, comme nous l'avons dit dans notre exposé, en France on doit constater la force de l'opérateur ferroviaire national dans le domaine des voyageurs, où le défi a été relevé à bien des égards, alors que sa position est certainement moins dynamique du côté du fret ferroviaire. Je souhaite donc sur ce point recueillir l'avis du grand témoin Michel Walrave et celui de l'historien géographe qu'est Etienne Auphan.

\section{Michel Walrave}

Je ne dirai pas que la spécialisation est une innovation française, car les Japonais ont construit aussi une ligne spécialisée ; mais les Japonais devaient compter avec une caractéristique particulière, c'est que le réseau japonais existant était à voie étroite, ne permettant pas la grande vitesse, donc cette spécialisation à la grande vitesse s'est trouvée au Japon tout à fait naturelle, liée au fait qu'évidemment le volume des trafics à transporter et la nécessité d'avoir des plages d'entretien la nuit ne permettait pas d'envisager une utilisation de cette ligne pour le trafic marchandises. En Europe, je 
crois que ce qu'il faut bien voir c'est que les premières lignes qui ont été conçues en Allemagne et en Italie étaient des lignes non spécialisées et que c'est l'Espagne qui, après la France, a fait la première le choix de lignes spécialisées. Il y a plusieurs raisons à cela ; il y a une raison de sécurité, car faire coexister du trafic marchandises avec des trains à grande vitesse dans les mêmes plages horaires peut poser des problèmes - c'est moins vrai si on spécialise par périodes, les trains de marchandises circulant la nuit et les TGV de jour, mais la nuit il faut aussi ménager des plages pour l'entretien des voies. Cette spécialisation a surtout résulté en France de l'économie de construction que nous visions pour les lignes nouvelles puisqu'on a pu adopter ainsi des pentes beaucoup plus importantes, ce qui permettait d'éviter tunnels et ouvrages d'art dans une large mesure. En Europe, progressivement, le choix de la spécialisation, je ne dirai pas s'est généralisé, mais s'est imposé de manière assez large. Les nouvelles lignes allemandes sont des lignes spécialisées, les nouvelles lignes italiennes également et cette idée de la spécialisation, du fait de ses avantages, à l'exemple français et espagnol, a gagné du terrain. Il faut aussi souligner qu'on peut adapter les caractéristiques géométriques de la voie - j'entre un peu dans la technique -, les rayons de courbure, les dévers à la vitesse des trains, cette vitesse étant homogène ; il en résulte des usures de voie beaucoup moins importantes que sur une ligne appelée à supporter des trains dont les charges par essieu et les vitesses sont hétérogènes. Enfin, le réseau français étant déjà relativement maillé, l'idée était qu'en adoptant cette spécialisation on pouvait libérer de la capacité sur les lignes existantes pour le trafic marchandises. Cette spécialisation n'est pas non plus un dogme absolu, on le voit avec la ligne nouvelle vers l'Espagne par Barcelone qui sera une ligne mixte pour donner des débouchés à l'écartement européen au port de Barcelone ; ce sera la même chose pour le "Y basque " pour le port de Bilbao. Sur les lignes où la densité du trafic permet la coexistence de ces deux types de trafic, je crois qu'on peut très bien envisager des lignes mixtes. Il y a aussi un débat à propos de la branche sud du Rhin-Rhône, pour savoir si cela doit être une ligne spécialisée ou mixte. Je crois que tous ces débats sont légitimes, il s'agit simplement de mettre en balance avantages et inconvénients des solutions de la spécialisation et de la mixité. Je crois que cette spécialisation s'imposait sur le réseau TGV français pour toutes les lignes radiales au départ de Paris, elle s'impose peut-être moins sur d'autres axes, comme par exemple le cas du tunnel sous la Manche, ou l'intêret de la sécurité est évident, ou celui du Lyon-Turin où la spécialisation serait une absurdité ; dans toutes ces débats il faut raison garder et mener une analyse économique rigoureuse pour déterminer avantages et inconvénients des deux solutions.

\section{Etienne Auphan}

Deux observations : par comparaison avec l'Espagne et avec le Japon, la France est le seul pays parmi les trois où ne se posait pas au départ le problème d'un écartement différent, voie étroite au Japon, vous l'avez dit, ou voie large en Espagne. La situation française est donc un peu particulière. Ensuite, à propos de cet argument que vous avez rappelé, et qui a été longtemps avancé, la spécialisation des lignes nouvelles à grande vitesse aux voyageurs permet de dégager des sillons sur le réseau classique pour le fret : en fait on s'est heurté à deux facteurs, deux oppositions qui l'ont contredite. Entre temps s'est produite en effet la régionalisation des services ferroviaires et le développement des TER suburbains et périurbains si bien que l'acheminement des trains de fret se trouve en discordance, en opposition dans les grandes métropoles, avec le trafic TER. La seconde c'est que, précisément, les anciennes voies ferrées, les voies ferrées classiques construites au $\mathrm{XIX}^{\mathrm{e}}$ siècle, ont pour caractéristique de traverser 
aujourd'hui les centres villes et les agglomérations qui se sont développées autour d'elles, et sont source de toutes les nuisances que cela peut entraîner. On sait évidemment qu'un train de fret lourd engendre des nuisances considérables sur le plan sonore alors que la majorité du trafic se fait de nuit. D'où la nécessité de construire des déviations des grandes agglomérations, voir les exemples et les projets de Lyon et de Bordeaux, mais là on se heurte à quelque chose de tout à fait inconnu, si je puis dire, en France : investir sur le rail dans quelque chose de bien moins spectaculaire et populaire que les lignes à grande vitesse Paris-province et pour lequel la priorité budgétaire et l'acceptation sociale apparaissent beaucoup plus problématiques. Il est donc plus difficile d'investir lourdement dans des contournements de grandes agglomérations. Je sais bien que la déviation de Tours a été profilée de telle manière qu'elle puisse recevoir du trafic de fret mais il reste un certain nombre d'exemples en France où la situation est beaucoup plus complexe à résoudre.

\section{Gabriel Dupuy}

Voici venu le moment de notre dernière et commune question à Michel Walrave : vous avez entendu le bilan des travaux de l'AHICF et notre programme de recherche, comment, en tant que témoin, y réagissez-vous?

\section{Michel Walrave}

Vous me posez une question difficile. Dans toute la fresque brossée par le professeur Auphan, peut-être a-t-il été donné une place disproportionnée aux services voyageurs par rapport aux services marchandises, bien que ça soit à mon avis un sujet extrêmement difficile ; l'analyse de l'économie et de l'impact sur l'aménagement de l'évolution des installations des lignes ouvertes au trafic marchandises est encore plus difficile à mener que pour celles ouvertes aux voyageurs, mais c'est un sujet qui n'a pas été beaucoup défriché. Pour prendre dans l'histoire plus récente un exemple sur un sujet que je connais bien, je crois qu'un thème sur lequel il serait tout à fait intéressant de mobiliser des jeunes chercheurs, c'est l'utilisation des opportunités qu'a représentée la création des nouvelles gares liées au réseau à grande vitesse pour le développement régional et local, parce qu'on constate là une grande diversité de situations. Par exemple, à Lille, l'occasion de l'arrivée du TGV Nord et de la nouvelle gare de LilleEurope a été vraiment parfaitement mise à profit par les autorités régionales et locales pour en faire un grand pôle de développement. À l'inverse, il ne s'est rien passé autour de la gare de Mâcon, alors qu'on aurait pu penser que ç'aurait pu être une opportunité pour l'agglomération mâconnaise ; si je prends la gare de l'Arbois, c'est-à-dire d'Aix-enProvence TGV, elle va conduire à créer un pôle de développement extrêmement important, même si son utilité avait été contestée par le maire de Marseille de l'époque qui avait une vision très (trop ?) marseillaise des choses... Ce sujet de l'impact et de l'utilisation de l'opportunité que représente la création d'une gare nouvelle pour le développement serait un thème intéressant ; il y aurait certainement bien d'autres thèmes, tels ceux évoqués par le professeur Dupuy.

\section{Gabriel Dupuy}

À la fin de cette table ronde, je tiens à remercier tout particulièrement Michel Walrave, qui nous a donné des éclairages originaux et des suggestions qui seront notées sur l'agenda de l'AHICF. Etienne Auphan et moi rendons hommage à Maurice Wolkowitsch, membre fondateur de l'AHICF et membre de son comité scientifique pendant plus de quinze ans, qui aurait dû, s'il n'avait été empêché aujourd'hui, présider cette séance à notre place. 


\section{NOTES}

*. Le programme du colloque comprenait une intervention de M. Kyung Chul Lee, «Grandes vitesses françaises et coréennes ».

\section{RÉSUMÉS}

Ce débat entre Etienne Auphan, professeur émérite à l'université de Paris-Sorbonne et Michel Walrave, directeur général honoraire de l'Union internationale des chemins de fer, animé par Gabriel Dupuy, professeur à l'université de Paris I - Panthéon-Sorbonne, met en perspective les interventions précédentes, tant sur le bilan des vingt ans d'études menées par l'AHICF que sur les expériences de pays étrangers dans le domaine de la grande vitesse ferroviaire. La première question posée par G. Dupuy est celle de l'adaptation d'un système de grande vitesse ferroviaire à un territoire donné, à travers les exemples précédemment développés. M. Walrave commente les différences entre réseaux à grande vitesse français, coréen et espagnol. E. Auphan pense pour sa part que le modèle français de réseau à grande vitesse a été conçu dans le cadre d'un territoire déterminé et qu'on ne peut pas l'en séparer. Son adaptation à d'autres territoires est donc la question qui reste posée et qui n'a sans doute pas été suffisamment prise en compte lors de l'exportation de la technologie française.

La deuxième question posée est celle de l'interconnexion au sein des réseaux ferroviaires à grande vitesse par des gares de passage de leurs trains dans le cœur de la ville, comme à Madrid et à Barcelone, par opposition avec les choix français d'interconnexion autour de Paris. M. Walrave remarque que ce choix assure la complémentarité entre transport aérien et transport ferroviaire à Roissy - Charles-de-Gaulle. Pour E. Auphan, ce contournement de l'île-de-France est ambigu car, résultat de la conjonction de divers intérêts (aéroport, Eurodisney...), l'interconnexion donne à la fois la possibilité d'assurer des relations de province à province transversales et la desserte périphérique des pôles d'activité parisiens, renforçant donc leur concentration.

La troisième question est celle de la spécialisation des réseaux à grande vitesse pour les trafics de marchandises et de voyageurs, différente selon les pays. Pour M. Walrave, le choix français de la spécialisation des trafics s'est imposé en Europe avec raison, en particulier en France pour les lignes radiales pour des raisons de tracé et de densité des circulations. En revanche, seule une analyse économique rigoureuse pourra déterminer les avantages et les inconvénients des deux solutions pour les lignes à venir. E. Auphan met en valeur les problèmes que cause l'acheminement du trafic fret, en particulier dans les agglomérations, et qui contredisent l'argument selon lequel la spécialisation des lignes nouvelles à grande vitesse pour les voyageurs permet de dégager des sillons sur le réseau classique pour le fret.

La réaction de $\mathrm{M}$. Walrave, en tant que témoin, au bilan des travaux de l'AHICF et à son programme de recherche clôt le débat.

This debate between Etienne Auphan, professor emeritus at the University of Paris-Sorbonne and Michel Walrave, honorary general manager of the Union i nternationale des chemins de fer, moderated by Gabriel Dupuy, professor at the university of Paris I Panthéon-Sorbonne, puts the 
preceding papers into a critical perspective, in relation to an assessment of the past twenty years of studies led by the AHICF as well in connection with the experiences of foreign countries in the field of high-speed railways. G. Dupuy's first question concerned the adaptation of a high-speed railway to a specific territory, through previously developed examples. Mr. Walrave commented on the differences between French, Korean, and Spanish high-speed networks. E. Auphan was of the mind that the model of the French high-speed network was conceived in the context of a specific territory and cannot be considered separately. Its adaptability to other areas remains in question and has definitely not been taken sufficiently into account when French technology has been exported.

The second question concerns the interconnection of trains at the core of high-speed railway networks via transit stations in the center of a city, as in Madrid and Barcelona, versus the French choice of locating interconnections around rather than in Paris. Mr. Walrave observed that this choice ensures the complementarity of air and rail transportation at Roissy-Charles-deGaulle. The bypass through the Île-de-France is multifaceted for E. Auphan, since the interconnection, which is the result of the intersection of various points of interest (airport and Euro Disney, for instance), creates the possibility both of providing cross-relationships from province to province and servicing the periphery of the poles of Parisian activity, thereby reinforcing their concentration.

The third question concerns the specialization of high-speed networks for traffic in merchandise and passengers, which varies from country to country. For Mr. Walrave, the specialization of traffic impressed itself appropriately upon Europe, especially in France with respect to radial lines, for reasons related to routes and density of circulation.

E. Auphan emphasized problems caused by the routing of freight traffic, especially in built-up areas, contradicting the argument that the specialization of new high-speed lines for travelers leaves lines in the traditional network free for freight.

Mr. Walrave's reaction as a witness to the assessment of the AHICF's work and research program ended the debate.

\section{INDEX}

Mots-clés : grande vitesse, Espagne, Corée, réseau, aménagement du territoire, politique des transports/International

Keywords : high speed, Spain, Korea, network, town and country planning, transportation policy/International

\section{AUTEURS}

\section{GABRIEL DUPUY}

Professeur à l'université de Paris I - Panthéon-Sorbonne, membre du Comité scientifique de l'AHICF

\section{ETIENNE AUPHAN}

Professeur émérite à l'université de Paris-Sorbonne (Paris IV), membre du Comité scientifique de l'AHICF

\section{MICHEL WALRAVE}

Directeur général honoraire de l'Union internationale des chemins de fer 